\title{
INSTRUMENTATION FOR THE CORNELL ERL INJECTOR TEST CRYOSTATS*
}

\author{
P. Quigley ${ }^{\dagger}$, S. Belomestnykh, M. Liepe, V. Medjidzade, J. Sears, V. Veshcherevich, \\ Laboratory for Elementary-Particle Physics, Cornell University, Ithaca, NY 14853, U.S.A.
}

\section{Abstract}

Cornell is building a $1.3 \mathrm{GHz}$ Injector Cryomodule for an ERL prototype. The cryomodule consists of five two-cell niobium cavities, each cavity having two coaxial input couplers. Cavity and coupler pairs require acceptance testing at high power prior to assembly in the injector cryomodule. A liquid nitrogen cryostat for testing the couplers at high power has been built and the first input coupler test is complete. In addition, a Horizontal Test Cryostat (HTC) is being built to test input coupler pairs and cavities as a set. The first HTC test is scheduled for summer 2007. Details for instrumentation of the Coupler Test Cryostat (CTC) and HTC are presented.

\section{INTRODUCTION}

The ERL Injector cryomodule will use coaxial input couplers to feed RF power to its five two-cell cavities. These couplers were tested in the high-power RF
Processing Area (PA) at Wilson Lab in a Coupler Test Cryostat (CTC) [1]. A Horizontal Test Cryostat (HTC) [2] is being built to test $1.3 \mathrm{GHz}$ two-cell RF structures, the previously tested coupler pair, two HOM loads [3], and the cryogenic cooling scheme (See Figure 1).

These cryostats are fitted with several different types of sensors for system monitoring and control. The RF PA has been equipped with a modular PLC test rack [4] that is configured to easily adapt to any cryostat similar in cryogenic function to the CESR cryomodules. Data is acquired through two principal means. The first consists of stand-alone signal conditioners with analog output to a Allen Bradley PLC. The PLC has an Ethernet module that sends data out to a subnet. The second is a commercial signal conditioning unit with an A/D converter and Ethernet interface. Both systems send data to EPICS [5] for control and display.

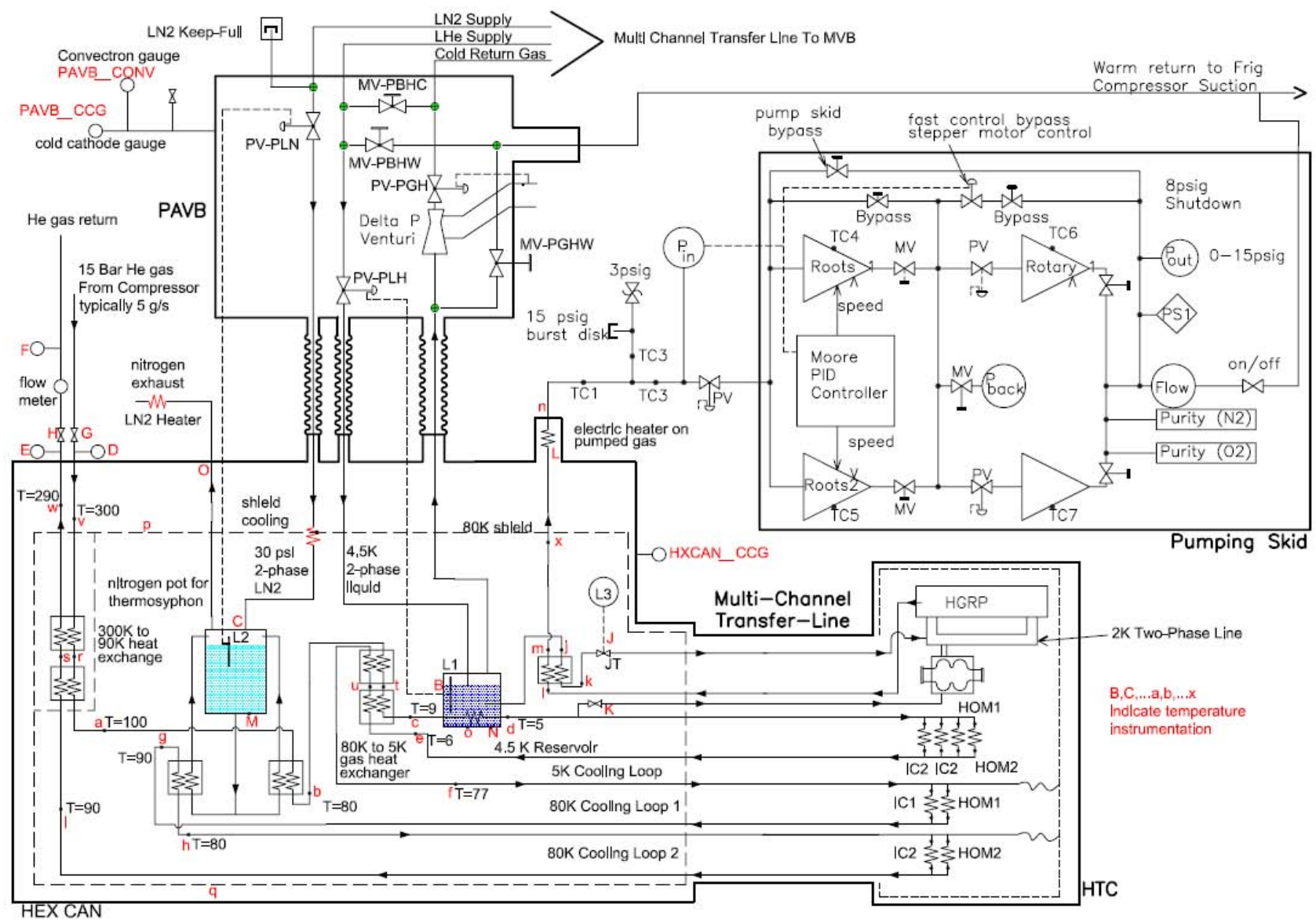

Figure 1: Cornell ERL injector cryogenic system diagram.

\footnotetext{
* Supported by the National Science Foundation grant PHY 0131508.

$\dagger$ †pq1@.cornell.edu
} 


\section{CRYOGENIC CONTROL}

The cryogens from a refrigeration and liquefaction plant are provided to the CTC, cryogenic heat-exchanger box (HEX CAN) and HTC via a cold Processing Area Valve Box (PAVB) designed for testing CESR RF cryomodules. The liquid nitrogen (LN2) valve body and stem were extended and seat plug changed to convert the valve from manual to proportional control. For the CTC, only LN2 is needed. The HEX CAN and HTC require both LN2 and liquid helium (LHe). The HEX CAN has LN2 and LHe reservoirs.

The LN2 level stick is a commercial capacitive type sensor while the LHe stick is a resistive device. Both use commercial controllers with $4-20 \mathrm{~mA}$ output sent to the PLC. PID loops in the PLC provide PAVB valve control and thus liquid level regulation. Cold helium gas return is operated in an open loop configuration.

The HTC takes cryogen supply and return via the HEX CAN. The system has a pre-cool circuit for slow cooldown via a manual valve. When the pre-cool is finished, the system switches to the normal helium loop.

The HTC has four $2 \mathrm{~K}$ LHe level sticks installed in the two-phase line; two of the four are spares. The $2 \mathrm{~K}$ helium level controllers send process variable (PV) signals to the PLC. The PLC output module control variable (CV) regulates the HEX CAN Joule-Thompson (JT) valve that supplies helium liquid to the HTC.

The HTC LHe level sticks look at both ends of the $2 \mathrm{~K}$ two-phase line. Helium level differences at the ends of this line may provide useful data for control optimization. A Helium cold Gas Return Pipe (HGRP) is part of the $2 \mathrm{~K}$ vacuum pumping line. The $2 \mathrm{~K}$ return gas passes through a counter-flow heat exchanger where it pre-cools the incoming $4 \mathrm{~K}$ helium just upstream of the JT valve. A resistive heater then warms the return gas to room temperature for a pumping skid. The operating pressure is between 23 and 12 Torr. The $2 \mathrm{~K}$ pressure control is independent of the PLC. This loop is regulated with a PID controller. The PV for this loop is a room temperature capacitive manometer, and the $\mathrm{CV}$ for the pumping skid is an AC motor speed control.

\section{CRYOMODULE SENSORS \& INSTRUMENTATION}

\section{Temperature Sensors}

Several types of temperature sensors are employed in these cryostats (see Table 1and Figure 2):

- CLTS (Cryogenic Linear Temperature Sensor)

- PT100, PRT (Platinum Resistance Thermometer)

- AB-RT (Allen Bradley 100 Ohm Carbon Resistor)

- TC (Thermocouple, T-type and K-type)

- IR Sensor (Infrared Sensor)
CLTS's are 4-wire temperature sensors with a near linear response from $\sim 300 \mathrm{~K}$ to $4 \mathrm{~K}$. CLTS response at $2 \mathrm{~K}$ will be tested with the HTC.

Table 1: List of temperature sensors.

\begin{tabular}{|l|l|c|}
\hline \multicolumn{1}{|c|}{ Sensor } & \multicolumn{1}{|c|}{$\begin{array}{c}\text { Temperature } \\
\text { Range }\end{array}$} & Where used \\
\hline CLTS & $2 \mathrm{~K}$ to $300 \mathrm{~K}$ & CTC, HTC \\
\hline PT100 & $73 \mathrm{~K}$ to $450 \mathrm{~K}$ & HTC \\
\hline AB-RT & $1.5 \mathrm{~K}$ to $20 \mathrm{~K}$ & HTC \\
\hline TC & $77 \mathrm{~K}$ to $450 \mathrm{~K}$ & CTC,HTC \\
\hline IR Sensor & $233 \mathrm{~K}$ to $873 \mathrm{~K}$ & CTC \\
\hline
\end{tabular}

PT100's are 4-wire temperature sensors that have a resistance of $100 \mathrm{Ohms}$ at $0^{\circ} \mathrm{C}$ and $134 \mathrm{Ohms}$ at $100^{\circ} \mathrm{C}$. These sensors will be used in the HTC to monitor RF coupler surfaces expected to operate above LN2 temperature. They will also be used as a PV for an in-situ coupler bake.

AB-RT's are 4-wire carbon resistor thermometers. These sensors are mounted on the HTC HGRP and inside the $2 \mathrm{~K}$ two-phase helium line.

Both CTC and HTC use T-type thermocouples on the RF input couplers. The HTC tuner motor windings have a K-type thermocouple.

The CTC uses IR sensors to monitor RF coupler center conductor temperature near the cold ceramic. The unit is mounted inside the insulation vacuum. The HTC is not equipped with IR sensors.

Sensors were both demountable and permanently mounted where practical for reuse on subsequent tests.

\section{RF Cables}

HTC cavity RF field probes, WPM (Wire Position Monitor), HOM field probes are ported out of the cryomodule via $0.86^{\prime \prime}$ diameter, stainless steel, semi-rigid coaxial cables with SMA connectors. Cable layout has carefully designed routes inside the $80 \mathrm{~K}$ shield. Right angle bends, and bulkhead connectors at the $80 \mathrm{~K}$ shield reduce connection count. Cable between $80 \mathrm{~K}$ and $300 \mathrm{~K}$ is 0.141 " diameter semi-flexible RF coax. Due to low radiation levels for the HTC, all RF cables in the HTC have PTFE dielectric.

\section{Cavity Tuner, Piezo and Motor}

The HTC has four piezo tuner actuators, each with an integral force sensor. The tuner motor is specifically designed for cryogenic operation and is driven with a compatible linear stepper motor drive.

\section{HTC Heaters}

The HTC is equipped with several heaters. The $2 \mathrm{~K}$ two-phase line has a 10 watt heater for calibration and load leveling. The HOM loads have 400 watt (80K loop) and 10 watt (5K loop) heaters for calibration. 


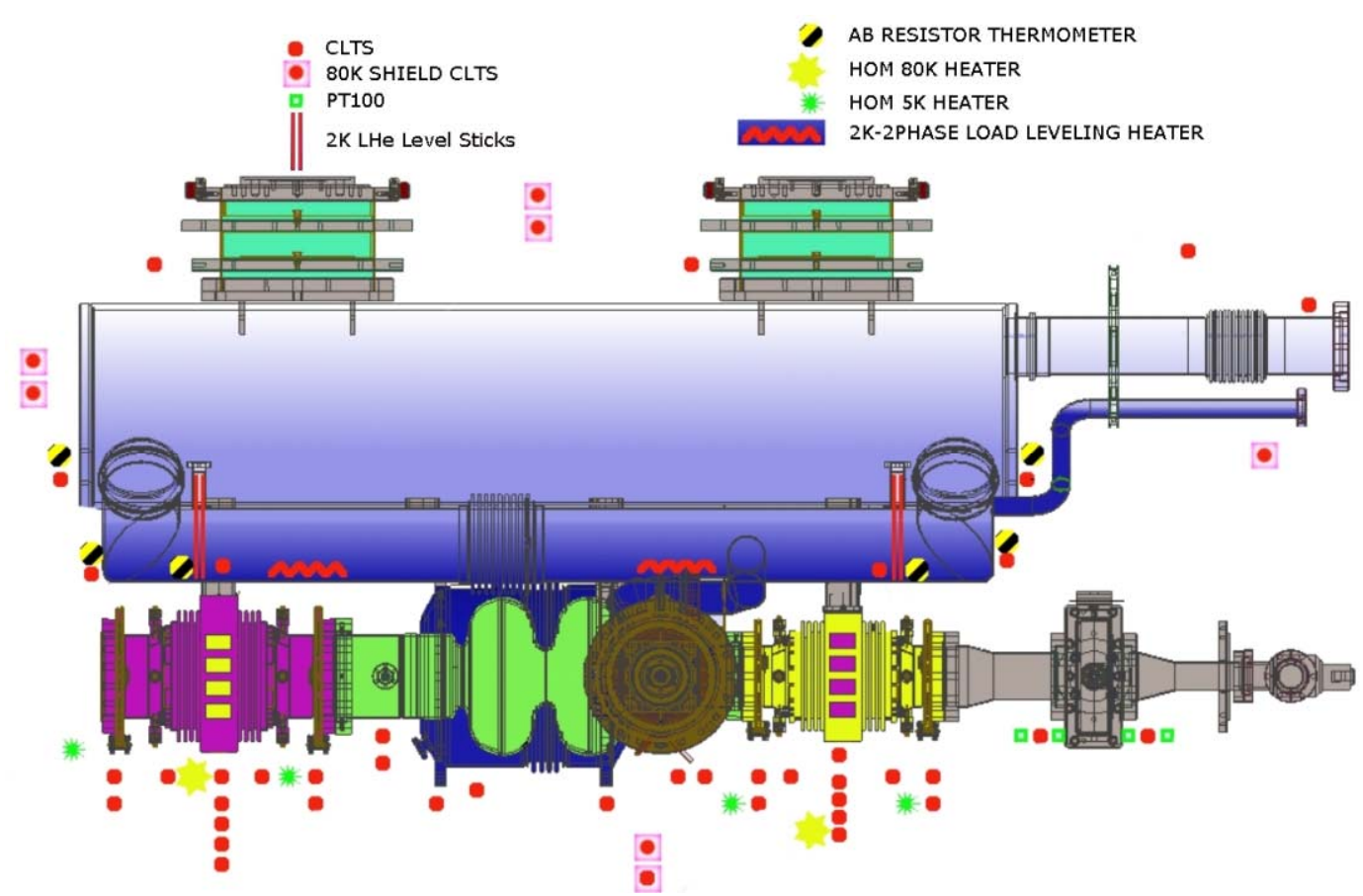

Figure 2: Temperature sensor map of HTC (input coupler sensors not shown).

\section{IN-SITU INPUT COUPLER BAKE}

For the HTC the plan is to bake the couplers in-situ with insulation vacuum established. Four Kapton ${ }^{\mathrm{TM}}$ film heaters will be mounted on each coupler, three inside the insulation vacuum space and one outside. Hot air will be blown into the coupler center conductor cooling port. As the cavity and tuner motor cannot get much hotter than $30^{\circ} \mathrm{C}$, cool gas will be forced through the $5 \mathrm{~K}$ circuit. The $2 \mathrm{~K}$ circuit will be purged with nitrogen.

\section{DATA ACQUISITION AND USER INTERFACE}

CTC data acquisition was done by modifying the CESR Digital Cryogenic control system [4] installed for CESR cryomodule testing. A breakout box was designed and built to take advantage of the built in modularity of the digital control system. The interface between the breakout box and the PLC rack remained unchanged.

The HTC uses the same data acquisition equipment as the CTC with some additions and modifications. The Sensoray 2519DIN is a sixteen channel A/D converter with an imbedded Ethernet interface along with a combination of commercial and in-house built signal conditioners. The A/D converter takes 4-wire sensor inputs and ports conditioned signals directly to the lab subnet for viewing, in this case, with EPICS. To avoid potential network problems, all control loops are hardwired to the PLC.

\section{CONCLUSIONS}

The in-situ coupler bake, if successful, will reduce cryomodule assembly and conditioning lead time. The CTC and HTC provided a window into the challenges of getting a cryomodule instrumented from sensor to displaying of acquired data. This information will prove useful when designing the same system for the Cornell ERL Injector Cryomodule and beyond.

\section{ACKNOWLEDGMENTS}

Authors would like to acknowledge the extensive effort put forth by the CESR/ERL Cryogenics Group: Richard Ehrlich, Eric N. Smith, Dan Sabol, Dwight Widger, and Colby Shore to get the HEX CAN designed and fabricated. Also this project would not have been possible without the expert help of the CLASSE technical shops and staff.

\section{REFERENCES}

[1] V. Veshcherevich, et al, "High Power Test of First Input Couplers for Cornell ERL Injector Cavities," this conference.

[2] M. Liepe, et al, "The Cornell ERL Superconducting 2Cell Injector Cavity String and Test Cryomodule," this conference.

[3] V. Shemelin, et al, "Status of HOM load for the

Cornell ERL Injector," EPAC'06, Edinburgh, June 2006, p. 478.

[4] P.Quigley, et al, "Digital Cryogenic Control System for Superconducting RF Cavities in CESR" PAC'03

Portland, May 2003, p. 1476.

[5] http://www.aps.anl.gov/epics/index.php

T04 Accelerator/Storage Ring Control Systems 